\title{
Risk Comparison of Improved Estimators in a Linear Regression Model with Multivariate $t$ Errors under Balanced Loss Function
}

\author{
Guikai Hu, ${ }^{1,2}$ Qingguo Li, ${ }^{1}$ and Shenghua $\mathrm{Yu}^{3}$ \\ ${ }^{1}$ School of Mathematics and Econometrics, Hunan University, Changsha 410082, China \\ ${ }^{2}$ School of Science, East China Institute of Technology, Nanchang 330013, China \\ ${ }^{3}$ School of Economics and Trade, Hunan University, Changsha 410079, China \\ Correspondence should be addressed to Qingguo Li; liqingguoli@yahoo.com.cn
}

Received 10 January 2014; Accepted 8 April 2014; Published 6 May 2014

Academic Editor: Francesco Pellicano

Copyright (c) 2014 Guikai Hu et al. This is an open access article distributed under the Creative Commons Attribution License, which permits unrestricted use, distribution, and reproduction in any medium, provided the original work is properly cited.

Under a balanced loss function, we derive the explicit formulae of the risk of the Stein-rule (SR) estimator, the positive-part Steinrule (PSR) estimator, the feasible minimum mean squared error (FMMSE) estimator, and the adjusted feasible minimum mean squared error (AFMMSE) estimator in a linear regression model with multivariate $t$ errors. The results show that the PSR estimator dominates the SR estimator under the balanced loss and multivariate $t$ errors. Also, our numerical results show that these estimators dominate the ordinary least squares (OLS) estimator when the weight of precision of estimation is larger than about half, and vice versa. Furthermore, the AFMMSE estimator dominates the PSR estimator in certain occasions.

\section{Introduction}

In the literature, many statisticians have studied the risk comparisons of various estimators in the linear model with normal errors and have generated substantial results. However, the assumption of normality restricts the range of possible applications. The multivariate $t$ distributions are more realistic and accurate than multivariate normal distributions in modeling real-word data due to their heavy tails. Moreover, multivariate $t$ distribution plays an important role in robust statistical inference. Therefore, various inference problems based on these distributions have been studied. The sampling performance of estimators is an important aspect among them.

Let us now consider a linear regression model

$$
y=X \beta+u,
$$

where $y$ is an $n \times 1$ vector of observations on a dependent variable. $X$ is an $n \times k$ full rank matrix of observations. $\beta$ is a $k \times 1$ vector of coefficients. We assume that $u$ has a multivariate $t$ distribution with the probability density function given by

$$
P(u \mid \alpha, \sigma)=\frac{g(\alpha)}{\left(\sigma^{2}\right)^{n / 2}} \frac{1}{\left(\alpha+u^{\prime} u / \sigma^{2}\right)^{(n+\alpha) / 2}},
$$

where $g(\alpha)=\alpha^{\alpha / 2} \Gamma((\alpha+n) / 2) / \pi^{n / 2} \Gamma(\alpha / 2)$. It is well known that its mean vector and covariance matrix are given by

$$
\begin{gathered}
E(u)=0, \quad \text { for } \alpha>1, \\
E\left(u u^{\prime}\right)=\frac{\alpha \sigma^{2}}{(\alpha-2)} I_{n}, \quad \text { for } \alpha>2 .
\end{gathered}
$$

As is shown in Zellner [1], the multivariate $t$ distribution can be viewed as a mixture of multivariate normal and inverted gamma distributions:

$$
P(u \mid \alpha, \sigma)=\int_{0}^{\infty} P_{N}(u \mid \tau) \cdot P_{I G}(\tau \mid \alpha, \sigma) d \tau,
$$


where

$$
\begin{gathered}
P_{N}(u \mid \tau)=\left(2 \pi \tau^{2}\right)^{-n / 2} \exp \left(-\frac{u u^{\prime}}{2 \tau^{2}}\right), \\
P_{I G}(\tau \mid \alpha, \sigma)=\frac{2\left(\alpha \sigma^{2} / 2\right)^{\alpha / 2}}{\Gamma(\alpha / 2)} \cdot \tau^{-(\alpha+1)} \exp \left(-\alpha \cdot \frac{\sigma^{2}}{2 \tau^{2}}\right) .
\end{gathered}
$$

The ordinary least squares (OLS) estimator of $\beta$ is $b=S^{-1} X^{\prime} y$, where $S=X^{\prime} X$. Also, the Stein-rule (SR) estimator is

$$
b_{\mathrm{SR}}=\left(1-\frac{a e^{\prime} e}{b^{\prime} S b}\right) b,
$$

where $e=y-X b, v=n-k$, and $a$ is a constant such that $0 \leq a \leq 2(k-2) /(v+2)$. Under the mean squared error of prediction, Stein [2] and James and Stein [3] proved that the SR estimator dominates the OLS estimator when the numbers of explanatory variables are more than two and the MSE of the SR estimator is minimized if $a=(k-2) /(v+2)$. Thus, we use this value of $a$ hereafter. From then on, lots of improved estimators have been proposed. For example, Baranchik [4] proposed the positive-part Stein-rule (PSR) estimator defined as

$$
b_{\mathrm{PSR}}=\max \left[0,1-\frac{a e^{\prime} e}{b^{\prime} S b}\right] b .
$$

Farebrother [5] proposed the feasible minimum mean squared error (FMMSE) estimator which is

$$
b_{\mathrm{FMMSE}}=\left(\frac{b^{\prime} S b}{b^{\prime} S b+e^{\prime} e / v}\right) b .
$$

Further, Ohtani [6] extended the FMMSE estimator to the adjusted feasible minimum mean squared error (AFMMSE) estimator by adjusting the degrees of the freedom of the component of the FMMSE estimator. The AFMMSE estimator is

$$
b_{\mathrm{AFMMSE}}=\left(\frac{b^{\prime} S b / k}{b^{\prime} S b / k+e^{\prime} e / v}\right) b
$$

Some results related to the comparisons of these estimators have been established. For example, Giles [7] considered the pretest estimator for linear restrictions. Namba [8] studied the PMSE performance of the biased estimators in a regression model when relevant regressors are omitted. Namba and Ohtani [9] gave the risk comparison of the Stein-rule estimator under the Pitman nearness criterion. There is a common characteristic in their studies. That is, the used loss functions were the quadratic function and its variants. However, in regression analysis, we are often interested in using an estimator which has high precision of estimation and high goodness of fit of model. In this situation, Zellner [10] proposed a balanced loss function which takes account of both precision of estimation and goodness of fit. Balanced loss function is a more comprehensive and reasonable standard than quadratic loss and residual sum of squares. Much work has been done about the balanced loss risk comparisons of improved estimators in the normal linear model. Some examples are Giles et al. [11], Ohtani et al. [12], Ohtani [13], and so on. Their results show that SR estimator is not admissible and is dominated by PSR estimator. However, do the conclusions still hold under multivariate $t$ errors and balanced loss function? And, do these estimators still dominate the OLS estimator? It is interesting to discuss them under multivariate $t$ distributions and balanced loss function. Thus, we will give the explicit formulae for the balanced loss risk of these estimators and compare their sampling performance by theoretical and numerical analysis. In the next section, the explicit formulae of balanced loss risk of these estimators are derived. In Section 3, we compare the risk performance by numerical evaluations. The proofs of main results are given in Section 4.

\section{Balanced Loss Function and Risk}

In order to discuss the performance of considered estimators, we consider the balanced loss function as

$$
\begin{aligned}
L(\widetilde{\beta}, \beta)= & \theta(y-X \widetilde{\beta})^{\prime}(y-X \widetilde{\beta})+(1-\theta) \\
& \times(X \widetilde{\beta}-X \beta)^{\prime}(X \widetilde{\beta}-X \beta),
\end{aligned}
$$

where $\theta$ is a scalar such that $0 \leq \theta \leq 1$, and $\widetilde{\beta}$ is any estimator of $\beta$. The corresponding risk function is $R(\widetilde{\beta})=E[L(\widetilde{\beta}, \beta)]$. Since $u$ has a multivariate $t$ distribution which can be viewed as the mixture of multivariate normal and inverted gamma distribution, we have

$$
R(\widetilde{\beta})=E[L(\widetilde{\beta}, \beta)]=E_{\tau} E[L(\widetilde{\beta}, \beta) \mid \tau] .
$$

If the null hypothesis is $H_{0}: \beta=0$ and the alternative is $H_{1}: \beta \neq 0$, then the test statistic for $H_{0}$ is $F=\left(b^{\prime} S b / k\right) /\left(e^{\prime} e / v\right)$. In the same way as that of Namba [8], we consider the general pretest estimator as

$$
\widehat{\beta}=I(F \geq c)\left(1+\gamma \frac{e^{\prime} e}{b^{\prime} S b}\right)^{\omega} b,
$$

where $I(A)$ is an indicator function such that $I(A)=1$ if an event $A$ occurs and $I(A)=0$ otherwise. $c$ is the critical value of the pretest, and $\omega$ is an arbitrary integer. The term $\widehat{\beta}$ reduces to the SR estimator when $c=0, \gamma=-a$, and $\omega=1$, and it reduces to the PSR estimator when $c=a v / k, \gamma=-a$, and $\omega=1$. Furthermore, $\widehat{\beta}$ reduces to the FMMSE estimator when $c=0, \gamma=1 / v$, and $\omega=-1$, and it reduces to the AFMMSE estimator when $c=0, \gamma=k / v$, and $\omega=-1$, respectively.

To derive the formulae of $R(\widehat{\beta})$, we first compute $E[L(\widehat{\beta}, \beta) \mid \tau]$, assuming that $\tau$ is given. If we denote $u_{1}=$ $b^{\prime} S b / \tau^{2}, u_{2}=e^{\prime} e / \tau^{2}$, then $u_{1} \sim \chi_{k}^{2}\left(\lambda_{1}\right)$, and $u_{2} \sim \chi_{n-k}^{2}$ for given $\tau$, where $\lambda_{1}=\beta^{\prime} S \beta / \tau^{2}, \chi_{f}^{2}(\lambda)$ is the noncentral chi-square distribution with $f$ degrees of freedom and noncentrality 
parameter $\lambda$. Thus, using $u_{1}$ and $u_{2}$, we define the functions $H(p, q, \gamma, c)$ and $J(p, q, \gamma, c)$ as

$$
\begin{aligned}
& H(p, q, \gamma, c)=E\left[I\left(\frac{v}{k} \cdot \frac{u_{1}}{u_{2}} \geq c\right)\left(\frac{u_{1}+\gamma u_{2}}{u_{1}}\right)^{p} u_{1}^{q} \mid \tau\right], \\
& J(p, q, \gamma, c) \\
& \quad=E\left[I\left(\frac{v}{k} \cdot \frac{u_{1}}{u_{2}} \geq c\right)\left(\frac{u_{1}+\gamma u_{2}}{u_{1}}\right)^{p} u_{1}^{q} \frac{\beta^{\prime} S b}{\tau^{2}} \mid \tau\right],
\end{aligned}
$$

where $p, q$ are arbitrary integers. By direct computation, we have

$$
\begin{aligned}
& E[L(\widehat{\beta}, \beta) \mid \tau] \\
& =\theta\left[\beta^{\prime} S \beta+n \tau^{2}-2 \tau^{2} H(\omega, 1, \gamma, c)+\tau^{2} H(2 \omega, 1, \gamma, c)\right] \\
& \quad+(1-\theta)\left[\tau^{2} H(2 \omega, 1, \gamma, c)-2 \tau^{2} J(\omega, 0, \gamma, c)+\beta^{\prime} S \beta\right] .
\end{aligned}
$$

In the following, we first give one lemma in order to obtain the explicit formulae of risk.

Lemma 1. The explicit formulae of $H(p, q, \gamma, c)$ and $J(p, q, \gamma, c)$ are

$$
\begin{aligned}
H(p, q, \gamma, c) & =\sum_{i=0}^{\infty} w_{i}\left(\lambda_{1}\right) G_{i}(p, q, \gamma, c), \\
J(p, q, \gamma, c) & =\lambda_{1} \sum_{i=0}^{\infty} w_{i}\left(\lambda_{1}\right) G_{i+1}(p, q, \gamma, c),
\end{aligned}
$$

where $G_{i}(p, q, \gamma, c)=\left(2^{q} \Gamma(n / 2+i+q) / \Gamma(k / 2+\right.$ i) $\Gamma(v / 2)) \int_{\mathcal{c}^{*}}^{1} t^{k / 2+i+q-p-1}(1-t)^{v / 2-1}[\gamma+(1-\gamma) t]^{p} d t$, $w_{i}(\lambda)=\exp (-\lambda / 2)(\lambda / 2)^{i} / i !$, and $c^{*}=k c /(k c+v)$.

By this lemma and (11) and (14), we have the following theorem.

Theorem 2. Under model (1) and loss function (10), the risk of the general pretest estimator $\widehat{\beta}$ is

$$
\begin{aligned}
R(\widehat{\beta})= & \beta^{\prime} S \beta+n \theta \sigma^{2} \frac{\alpha}{\alpha-2}+\frac{\sigma^{2}}{2} \frac{\alpha^{\alpha / 2}}{\Gamma(\alpha / 2)} \\
& \times \sum_{i=0}^{\infty} \frac{G_{i}(2 \omega, 1, \gamma, c)}{i !} \frac{\Gamma(\alpha / 2+i-1) \theta_{1}^{i}}{\left(\theta_{1}+\alpha\right)^{\alpha / 2+i-1}} \\
& -\theta \sigma^{2} \frac{\alpha^{\alpha / 2}}{\Gamma(\alpha / 2)} \sum_{i=0}^{\infty} \frac{G_{i}(\omega, 1, \gamma, c)}{i !} \frac{\Gamma(\alpha / 2+i-1) \theta_{1}^{i}}{\left(\theta_{1}+\alpha\right)^{\alpha / 2+i-1}} \\
& -2(1-\theta) \sigma^{2} \frac{\alpha^{\alpha / 2}}{\Gamma(\alpha / 2)} \\
& \times \sum_{i=0}^{\infty} \frac{G_{i+1}(\omega, 0, \gamma, c)}{i !} \frac{\Gamma(\alpha / 2+i) \theta_{1}^{i+1}}{\left(\theta_{1}+\alpha\right)^{\alpha / 2+i}} .
\end{aligned}
$$

According to this theorem, we can obtain the risk of SR, PSR, FMMSE, and AFMMSE estimators, respectively, and discuss their dominance properties. Firstly, we analyze the dominance properties between SR and PSR estimators.

Theorem 3. The PSR estimator dominates the SR estimator in terms of balanced loss risk when the error term of the model obeys a multivariate t distribution.

When the error term obeyed a multivariate normal distribution, Baranchik [4] proved that the PSR estimator dominated uniformly the SR estimator under the quadratic loss, and Ohtani [13] also proved that the SRSV estimator dominated uniformly the SR estimator under a balanced loss function. This theorem shows that when the loss function is extended to a balanced loss function, the dominance of the PSR estimator over the SR estimator still holds even if the error term obeys a multivariate $t$ distribution. This implies that the SR estimator is not admissible under a balanced loss function and multivariate $t$ errors.

Since further theoretical analysis of the risk of the SR, PSR, FMMSE, and AFMMSE estimators is difficult, we will compare them by numerical analysis in the next section.

\section{Numerical Analysis}

In order to compare the balanced loss risk of the estimators, we evaluated the values of relative risk defined by $\left(R(\widehat{\beta}) / \sigma^{2}\right) /\left(R(b) / \sigma^{2}\right)$. Thus, the estimator $\widehat{\beta}$ has smaller risk than the OLS estimator when the value of relative risk is smaller than unity. By Theorem 2, we can obtain the risks of the OLS, SR, PSR, FMMSE, and AFMMSE estimators, respectively. In the following, taking $\theta_{1}=\beta^{\prime} S \beta / \sigma^{2}$, the parameter values used in the numerical evaluations are $\theta_{1}=$ various values, $\theta=0,0.25,0.5,0.75,1, n=20,30,40, k=$ $3,5,8$, and $\alpha=3,5,7,10,20$. The numerical evaluations are executed on a personal computer using Version 7.9 (R2009b) MATLAB Software. In order to evaluate the integral in the risk expressions of these estimators, we use Trapezoidal method with 1000 equal subdivisions. Following the method used by Namba [8], the infinite series in these risk expressions is judged to converge when the increment of the infinite series becomes smaller than $10^{-12}$. Now, we give the relative risk of the SR, PSR, FMMSE, and AFMMSE estimators for the case of $n=20, k=5, \theta=0,0.25,0.5,0.75,1, \theta_{1}=$ $0,1,2,4,6,8,10,15,20$, and $\alpha=3,20$ in Tables 1 and 2 , respectively. According to Tables 1 and 2, it is sufficient to illustrate the result of Theorem 3. That is, the PSR estimator dominates the SR estimator under a balanced loss even if the error term obeys a multivariate $t$ distribution. We also find that when precision of estimation is more important (i.e., $\theta<0.5$ ), the SR and PSR estimators dominate the OLS estimator under the balanced loss function, and vice versa. This shows that the dominance of the SR and PSR estimators over the OLS estimator is not robust about the loss function. From Table 1, the FMMSE and AFMMSE estimators dominate the OLS estimator when the weight of precision of estimation is larger than about half, and vice versa. 
TABLE 1: Relative risk of the SR, PSR, FMMSE, and AFMMSE estimators for $n=20, k=5$ and $\alpha=3$.

\begin{tabular}{|c|c|c|c|c|c|}
\hline \multirow{2}{*}{$\theta_{1}$} & \multicolumn{5}{|c|}{$\theta$} \\
\hline & 0 & 0.25 & 0.5 & 0.75 & 1 \\
\hline & \multicolumn{5}{|c|}{ SR } \\
\hline 0 & 0.4705 & 0.8234 & 0.9999 & 1.1058 & 1.1764 \\
\hline 1 & 0.5015 & 0.8338 & 0.9999 & 1.0996 & 1.1661 \\
\hline 2 & 0.5262 & 0.8420 & 1.0000 & 1.0947 & 1.1579 \\
\hline 4 & 0.5640 & 0.8546 & 1.0000 & 1.0872 & 1.1453 \\
\hline 6 & 0.5923 & 0.8641 & 1.0000 & 1.0815 & 1.1358 \\
\hline 8 & 0.6148 & 0.8716 & 1.0000 & 1.0770 & 1.1284 \\
\hline 10 & 0.6333 & 0.8778 & 1.0000 & 1.0733 & 1.1222 \\
\hline 15 & 0.6686 & 0.8895 & 1.0000 & 1.0663 & 1.1104 \\
\hline \multirow[t]{2}{*}{20} & 0.6942 & 0.8980 & 1.0000 & 1.0611 & 1.1019 \\
\hline & \multicolumn{5}{|c|}{ PSR } \\
\hline 0 & 0.3533 & 0.7255 & 0.9116 & 1.0233 & 1.0977 \\
\hline 1 & 0.3918 & 0.7437 & 0.9196 & 1.0252 & 1.0955 \\
\hline 2 & 0.4231 & 0.7582 & 0.9258 & 1.0263 & 1.0934 \\
\hline 4 & 0.4715 & 0.7804 & 0.9349 & 1.0275 & 1.0893 \\
\hline 6 & 0.5079 & 0.7968 & 0.9413 & 1.0279 & 1.0857 \\
\hline 8 & 0.5367 & 0.8096 & 0.9461 & 1.0280 & 1.0826 \\
\hline 10 & 0.5603 & 0.8200 & 0.9499 & 1.0278 & 1.0798 \\
\hline 15 & 0.6050 & 0.8395 & 0.9567 & 1.0271 & 1.0740 \\
\hline \multirow[t]{2}{*}{20} & 0.6371 & 0.8533 & 0.9614 & 1.0262 & 1.0695 \\
\hline & \multicolumn{5}{|c|}{ FMMSE } \\
\hline 0 & 0.7254 & 0.8680 & 0.9394 & 0.9821 & 1.0107 \\
\hline 1 & 0.7391 & 0.8747 & 0.9425 & 0.9832 & 1.0104 \\
\hline 2 & 0.7503 & 0.8802 & 0.9451 & 0.9841 & 1.0101 \\
\hline 4 & 0.7680 & 0.8888 & 0.9492 & 0.9855 & 1.0096 \\
\hline 6 & 0.7816 & 0.8954 & 0.9523 & 0.9865 & 1.0092 \\
\hline 8 & 0.7926 & 0.9008 & 0.9548 & 0.9873 & 1.0089 \\
\hline 10 & 0.8019 & 0.9052 & 0.9569 & 0.9879 & 1.0086 \\
\hline 15 & 0.8197 & 0.9139 & 0.9609 & 0.9892 & 1.0080 \\
\hline \multirow[t]{2}{*}{20} & 0.8329 & 0.9202 & 0.9639 & 0.9901 & 1.0076 \\
\hline & \multicolumn{5}{|c|}{ AFMMSE } \\
\hline 0 & 0.3357 & 0.7042 & 0.8885 & 0.9991 & 1.0728 \\
\hline 1 & 0.3641 & 0.7186 & 0.8959 & 1.0022 & 1.0731 \\
\hline 2 & 0.3885 & 0.7308 & 0.9020 & 1.0047 & 1.0732 \\
\hline 4 & 0.4285 & 0.7508 & 0.9119 & 1.0086 & 1.0730 \\
\hline 6 & 0.4605 & 0.7666 & 0.9196 & 1.0114 & 1.0726 \\
\hline 8 & 0.4871 & 0.7795 & 0.9258 & 1.0135 & 1.0720 \\
\hline 10 & 0.5096 & 0.7905 & 0.9309 & 1.0152 & 1.0713 \\
\hline 15 & 0.5541 & 0.8119 & 0.9407 & 1.0180 & 1.0696 \\
\hline 20 & 0.5875 & 0.8277 & 0.9478 & 1.0198 & 1.0679 \\
\hline
\end{tabular}

This indicates that the dominance results of the FMMSE and AFMMSE estimators over the OLS estimator do not hold necessarily under the balanced loss function. It is easy to see that the risk of the AFMMSE estimator is much smaller than the risks of the SR and PSR estimators if $\theta<0.5$. However, the AFMMSE estimator does not dominate the FMMSE estimator under the balanced loss function when $\theta \geq 0.75$.
TABLE 2: Relative risk of the SR, PSR, FMMSE, and AFMMSE estimators for $n=20, k=5$ and $\alpha=20$.

\begin{tabular}{|c|c|c|c|c|c|}
\hline \multirow{2}{*}{$\theta_{1}$} & \multicolumn{5}{|c|}{$\theta$} \\
\hline & 0 & 0.25 & 0.5 & 0.75 & 1 \\
\hline & \multicolumn{5}{|c|}{ SR } \\
\hline 0 & 0.4705 & 0.8234 & 0.9999 & 1.1058 & 1.1764 \\
\hline 1 & 0.5537 & 0.8512 & 1.0000 & 1.0892 & 1.1487 \\
\hline 2 & 0.6176 & 0.8725 & 1.0000 & 1.0765 & 1.1274 \\
\hline 4 & 0.7074 & 0.9025 & 1.0000 & 1.0585 & 1.0975 \\
\hline 6 & 0.7662 & 0.9221 & 1.0000 & 1.0468 & 1.0779 \\
\hline 8 & 0.8068 & 0.9356 & 1.0000 & 1.0386 & 1.0644 \\
\hline 10 & 0.8362 & 0.9454 & 1.0000 & 1.0328 & 1.0546 \\
\hline 15 & 0.8823 & 0.9608 & 1.0000 & 1.0235 & 1.0392 \\
\hline \multirow[t]{2}{*}{20} & 0.9087 & 0.9696 & 1.0000 & 1.0183 & 1.0304 \\
\hline & \multicolumn{5}{|c|}{ PSR } \\
\hline 0 & 0.3533 & 0.7255 & 0.9116 & 1.0233 & 1.0977 \\
\hline 1 & 0.4568 & 0.7743 & 0.9331 & 1.0283 & 1.0918 \\
\hline 2 & 0.5386 & 0.8122 & 0.9490 & 1.0311 & 1.0858 \\
\hline 4 & 0.6560 & 0.8653 & 0.9699 & 1.0327 & 1.0745 \\
\hline 6 & 0.7329 & 0.8988 & 0.9818 & 1.0316 & 1.0648 \\
\hline 8 & 0.7852 & 0.9209 & 0.9888 & 1.0295 & 1.0566 \\
\hline 10 & 0.8220 & 0.9359 & 0.9929 & 1.0271 & 1.0499 \\
\hline 15 & 0.8771 & 0.9574 & 0.9976 & 1.0216 & 1.0377 \\
\hline \multirow[t]{2}{*}{20} & 0.9066 & 0.9682 & 0.9991 & 1.0176 & 1.0299 \\
\hline & \multicolumn{5}{|c|}{ FMMSE } \\
\hline 0 & 0.7254 & 0.8680 & 0.9394 & 0.9821 & 1.0107 \\
\hline 1 & 0.7621 & 0.8860 & 0.9479 & 0.9851 & 1.0099 \\
\hline 2 & 0.7914 & 0.9003 & 0.9547 & 0.9874 & 1.0091 \\
\hline 4 & 0.8348 & 0.9213 & 0.9646 & 0.9906 & 1.0079 \\
\hline 6 & 0.8648 & 0.9359 & 0.9714 & 0.9927 & 1.0069 \\
\hline 8 & 0.8864 & 0.9463 & 0.9762 & 0.9942 & 1.0062 \\
\hline 10 & 0.9025 & 0.9540 & 0.9798 & 0.9952 & 1.0055 \\
\hline 15 & 0.9288 & 0.9666 & 0.9855 & 0.9968 & 1.0044 \\
\hline \multirow[t]{2}{*}{20} & 0.9444 & 0.9740 & 0.9888 & 0.9977 & 1.0036 \\
\hline & \multicolumn{5}{|c|}{ AFMMSE } \\
\hline 0 & 0.3357 & 0.7042 & 0.8885 & 0.9991 & 1.0728 \\
\hline 1 & 0.4122 & 0.7429 & 0.9083 & 1.0075 & 1.0736 \\
\hline 2 & 0.4767 & 0.7752 & 0.9245 & 1.0140 & 1.0737 \\
\hline 4 & 0.5783 & 0.8255 & 0.9492 & 1.0234 & 1.0728 \\
\hline 6 & 0.6535 & 0.8622 & 0.9666 & 1.0292 & 1.0710 \\
\hline 8 & 0.7105 & 0.8896 & 0.9791 & 1.0328 & 1.0686 \\
\hline 10 & 0.7548 & 0.9105 & 0.9883 & 1.0350 & 1.0661 \\
\hline 15 & 0.8297 & 0.9447 & 1.0023 & 1.0368 & 1.0598 \\
\hline 20 & 0.8748 & 0.9645 & 1.0093 & 1.0361 & 1.0541 \\
\hline
\end{tabular}

In sum, our results show that when the loss function and error terms are extended from the usual quadratic loss function and normal distribution to balanced loss function and multivariate $t$ distribution, the dominance of the PSR estimator over the SR estimator is robust. However, the dominance of these estimators over the OLS estimator is not robust. 


\section{Proof of Main Results}

Proof of Lemma 1. For given $\tau, u_{1} \sim \chi_{k}^{2}\left(\lambda_{1}\right)$ and $u_{2} \sim \chi_{n-k}^{2}$; meanwhile, $u_{1}$ and $u_{2}$ are mutually independent. Therefore, we have

$$
\begin{aligned}
H(p, q, \gamma, c)= & E\left[I\left(\frac{v}{k} \frac{u_{1}}{u_{2}} \geq c\right)\left(\frac{u_{1}+\gamma u_{2}}{u_{1}}\right)^{p} u_{1}^{q} \mid \tau\right] \\
= & \sum_{i=0}^{\infty} w_{i}\left(\lambda_{1}\right) \frac{(1 / 2)^{n / 2+i}}{\Gamma(k / 2+i) \Gamma(v / 2)} \\
& \times \iint_{R}\left(u_{1}+\gamma u_{2}\right)^{p} u_{1}^{k / 2+i+q-p-1} u_{2}^{v / 2-1} \\
& \times \exp \left(-\frac{u_{1}+u_{2}}{2}\right) d u_{1} d u_{2},
\end{aligned}
$$

where $R$ is the region such that $(v / k)\left(u_{1} / u_{2}\right) \geq c$.

Making use of the change of variables, $v_{1}=u_{1} / u_{2}, v_{2}=$ $u_{2}$, the integral in (17) reduces to

$$
\begin{gathered}
\int_{k c / v}^{\infty} \int_{0}^{\infty} v_{1}^{k / 2+i+q-p-1} v_{2}^{n / 2+i+q-1}\left(v_{1}+\gamma\right)^{p} \\
\quad \times \exp \left(-\frac{v_{2}\left(1+v_{1}\right)}{2}\right) d v_{2} d v_{1} .
\end{gathered}
$$

Again, making use of the change of variables, $z=v_{2}\left(1+v_{1}\right) / 2$, $v_{1}=v_{1}$, the integral in (18) becomes

$$
\begin{aligned}
& 2^{n / 2+i+q} \Gamma\left(\frac{n}{2}+i+q\right) \\
& \quad \times \int_{k c / v}^{\infty} v_{1}^{k / 2+i+q-p-1}\left(v_{1}+\gamma\right)^{p}\left(1+v_{1}\right)^{-(n / 2+i+q)} d v_{1} .
\end{aligned}
$$

Further, making use of the change of a variable, $t=v_{1} /\left(1+v_{1}\right)$, the integral in (19) reduces to

$$
\int_{k c /(k c+v)}^{1} t^{k / 2+i+q-p-1}[\gamma+(1-\gamma) t]^{p}(1-t)^{v / 2-1} d t .
$$

By (17)-(20), we have

$$
H(p, q, \gamma, c)=\sum_{i=0}^{\infty} w_{i}\left(\lambda_{1}\right) G_{i}(p, q, \gamma, c) .
$$

Next, we derive the formula for $J(p, q, \gamma, c)$. Noting that $\partial \lambda_{1} / \partial \beta=2 S \beta / \tau^{2}$ and differentiating $H(p, q, \gamma, c)$ with respect to $\beta$, we have

$$
\begin{aligned}
\frac{\partial H(p, q, \gamma, c)}{\partial \beta}= & \sum_{i=0}^{\infty} \frac{\partial w_{i}\left(\lambda_{1}\right)}{\partial \beta} G_{i}(p, q, \gamma, c) \\
= & -\frac{S \beta}{\tau^{2}} H(p, q, \gamma, c) \\
& +\frac{S \beta}{\tau^{2}} \sum_{i=0}^{\infty} w_{i}\left(\lambda_{1}\right) G_{i+1}(p, q, \gamma, c) .
\end{aligned}
$$

Since $u_{1}=b^{\prime} S b / \tau^{2}$ and $b \sim N\left(\beta, \tau^{2}\left(X^{\prime} X\right)^{-1}\right), H(p, q, \gamma, c)$ can be expressed as

$$
H(p, q, \gamma, c)=\iint_{R}\left(\frac{u_{1}+\gamma u_{2}}{u_{1}}\right)^{p} u_{1}^{q} f_{1}(b) f_{2}\left(u_{2}\right) d u_{2} d b
$$

where $f_{2}\left(u_{2}\right)$ is the density function of $u_{2}$ and

$$
\begin{aligned}
f_{1}(b)= & \frac{1}{(2 \pi)^{k / 2}\left|\tau^{2}\left(X^{\prime} X\right)^{-1}\right|^{1 / 2}} \\
& \times \exp \left[-\frac{(b-\beta)^{\prime} X^{\prime} X(b-\beta)}{2 \tau^{2}}\right] .
\end{aligned}
$$

Differentiating (23) with respect to $\beta$, we have

$$
\begin{aligned}
& \frac{\partial H(p, q, \gamma, c)}{\partial \beta} \\
& =\iint_{R}\left(\frac{u_{1}+\gamma u_{2}}{u_{1}}\right)^{p} u_{1}^{q} f_{1}(b) f_{2}\left(u_{2}\right) \frac{S b-S \beta}{\tau^{2}} d u_{2} d b \\
& =\iint_{R}\left(\frac{u_{1}+\gamma u_{2}}{u_{1}}\right)^{p} u_{1}^{q} f_{1}(b) f_{2}\left(u_{2}\right) \frac{S b}{\tau^{2}} d u_{2} d b \\
& \quad-\frac{S \beta}{\tau^{2}} H(p, q, \gamma, c),
\end{aligned}
$$

which together with (22) yields that

$$
\begin{gathered}
\iint_{R}\left(\frac{u_{1}+\gamma u_{2}}{u_{1}}\right)^{p} u_{1}^{q} f_{1}(b) f_{2}\left(u_{2}\right) \frac{S b}{\tau^{2}} d u_{2} d b \\
=\frac{S \beta}{\tau^{2}} \sum_{i=0}^{\infty} w_{i}\left(\lambda_{1}\right) G_{i+1}(p, q, \gamma, c) .
\end{gathered}
$$

Multiplying $\beta^{\prime}$ from the left of the above, we have

$$
J(p, q, \gamma, c)=\lambda_{1} \sum_{i=0}^{\infty} w_{i}\left(\lambda_{1}\right) G_{i+1}(p, q, \gamma, c) .
$$

This completes the proof of this lemma.

Proof of Theorem 2. By Lemma 1, we have

$$
\begin{aligned}
& E_{\tau}\left[\tau^{2} H(p, q, \gamma, c)\right] \\
& =\sum_{i=0}^{\infty} G_{i}(p, q, \gamma, c) \int_{0}^{\infty} \tau^{2} w_{i}\left(\lambda_{1}\right) P_{I G}(\tau \mid \alpha, \sigma) d \tau \\
& =\sum_{i=0}^{\infty} \frac{G_{i}(p, q, \gamma, c)}{i !} \frac{\alpha^{\alpha / 2} \eta_{1}^{i}}{\Gamma(\alpha / 2)} 2^{1-\alpha / 2-i} \sigma^{\alpha} \\
& \quad \times \int_{0}^{\infty} \tau^{-(\alpha-1+2 i)} \exp \left(-\frac{\eta_{1}+\alpha \sigma^{2}}{2 \tau^{2}}\right) d \tau
\end{aligned}
$$


where $\eta_{1}=\beta^{\prime} S \beta$. Making use of the change of a variable, $t_{1}=$ $\left(\eta_{1}+\alpha \sigma^{2}\right) / 2 \tau^{2},(28)$ becomes

$$
\begin{aligned}
& \frac{\sigma^{2}}{2} \sum_{i=0}^{\infty} \frac{G_{i}(p, q, \gamma, c)}{i !} \frac{\alpha^{\alpha / 2}}{\Gamma(\alpha / 2)} \\
& \quad \times \Gamma\left(\frac{\alpha}{2}+i-1\right)\left(\frac{\eta_{1}}{\sigma^{2}}\right)^{i}\left(\frac{\eta_{1}}{\sigma^{2}}+\alpha\right)^{-\alpha / 2-i+1} .
\end{aligned}
$$

Taking $\theta_{1}=\eta_{1} / \sigma^{2},(29)$ becomes

$$
\frac{\sigma^{2}}{2} \frac{\alpha^{\alpha / 2}}{\Gamma(\alpha / 2)} \sum_{i=0}^{\infty} \frac{G_{i}(p, q, \gamma, c)}{i !} \frac{\Gamma(\alpha / 2+i-1) \theta_{1}^{i}}{\left(\theta_{1}+\alpha\right)^{\alpha / 2+i-1}}
$$

which together with (28) and (29) yields

$$
\begin{aligned}
E_{\tau}\left[\tau^{2} H(p, q, \gamma, c)\right]= & \frac{\sigma^{2}}{2} \frac{\alpha^{\alpha / 2}}{\Gamma(\alpha / 2)} \\
& \times \sum_{i=0}^{\infty} \frac{G_{i}(p, q, \gamma, c)}{i !} \frac{\Gamma(\alpha / 2+i-1) \theta_{1}^{i}}{\left(\theta_{1}+\alpha\right)^{\alpha / 2+i-1}}
\end{aligned}
$$

In a similar way, we have

$$
\begin{aligned}
& E_{\tau}\left[\tau^{2} J(p, q, \gamma, c)\right] \\
& =\sigma^{2} \frac{\alpha^{\alpha / 2}}{\Gamma(\alpha / 2)} \sum_{i=0}^{\infty} \frac{G_{i+1}(p, q, \gamma, c)}{i !} \frac{\Gamma(\alpha / 2+i) \theta_{1}^{i+1}}{\left(\theta_{1}+\alpha\right)^{\alpha / 2+i}}
\end{aligned}
$$

Obviously, we have $E_{\tau}\left(\tau^{2}\right)=\sigma^{2}(\alpha /(\alpha-2))$. This together with (11), (14), (31), and (32) yields the expression of $R(\widehat{\beta})$. The proof of this theorem is completed.

Proof of Theorem 3. By Theorem 2, let $\gamma=-a, \omega=1$; we have

$$
\begin{aligned}
R(\widehat{\beta})= & \beta^{\prime} S \beta+n \theta \sigma^{2} \frac{\alpha}{\alpha-2}+\frac{\sigma^{2}}{2} \frac{\alpha^{\alpha / 2}}{\Gamma(\alpha / 2)} \\
& \times \sum_{i=0}^{\infty} \frac{G_{i}(2,1,-a, c)}{i !} \frac{\Gamma(\alpha / 2+i-1) \theta_{1}^{i}}{\left(\theta_{1}+\alpha\right)^{\alpha / 2+i-1}} \\
& -\theta \sigma^{2} \frac{\alpha^{\alpha / 2}}{\Gamma(\alpha / 2)} \sum_{i=0}^{\infty} \frac{G_{i}(1,1,-a, c)}{i !} \frac{\Gamma(\alpha / 2+i-1) \theta_{1}^{i}}{\left(\theta_{1}+\alpha\right)^{\alpha / 2+i-1}} \\
& -2(1-\theta) \sigma^{2} \frac{\alpha^{\alpha / 2}}{\Gamma(\alpha / 2)} \\
& \times \sum_{i=0}^{\infty} \frac{G_{i+1}(1,0,-a, c)}{i !} \frac{\Gamma(\alpha / 2+i) \theta_{1}^{i+1}}{\left(\theta_{1}+\alpha\right)^{\alpha / 2+i}} .
\end{aligned}
$$

Since

$$
\begin{gathered}
\frac{\partial \int_{c^{*}}^{1} t^{k / 2+i+q-p-1}(1-t)^{v / 2-1}[\gamma+(1-\gamma) t]^{p} d t}{\partial c} \\
=-\left(\frac{k c}{k c+v}\right)^{k / 2+i+q-p-1}\left(1-\frac{k c}{k c+v}\right)^{v / 2-1} \\
\times\left[\gamma+(1-\gamma) \frac{k c}{k c+v}\right]^{p} \frac{k v}{(k c+v)^{2}},
\end{gathered}
$$

hence, differentiating (33) with respect to $c$ and performing some manipulations, we have

$$
\begin{aligned}
\frac{\partial R(\hat{\beta})}{\partial c}= & \sigma^{2} \frac{\alpha^{\alpha / 2}}{\Gamma(\alpha / 2)} \\
& \times \sum_{i=0}^{\infty} \frac{\Gamma(n / 2+i+1) \Gamma(\alpha / 2+i-1) \theta_{1}^{i}}{\Gamma(k / 2+i) \Gamma(v / 2)\left(\theta_{1}+\alpha\right)^{\alpha / 2+i-1} i !} \\
& \times \frac{(k c)^{k / 2+i-1} v^{v / 2-1}}{(k c+v)^{n / 2+i-1}} \times\left[-a+(1+a) \frac{k c}{k c+v}\right] \\
& \times\left\{-\left[-a+(1+a) \frac{k c}{k c+v}\right]+2 \theta \frac{k c}{k c+v}\right. \\
& \left.+2(1-\theta) \frac{k c}{k c+v} \frac{(\alpha / 2+i-1) \theta_{1}}{(k / 2+i)\left(\theta_{1}+\alpha\right)}\right\} .
\end{aligned}
$$

From (35), when $\gamma=-a, \omega=1$, a condition for $R(\widehat{\beta})$ to be monotonically decreasing is

$$
-a+(1+a) \frac{k c}{k c+v} \leq 0
$$

Thus, $R(\widehat{\beta})$ is monotonically decreasing on $c \in[0, a v / k]$ if $\gamma=-a, \omega=1$. Since $\widehat{\beta}$ becomes the SR estimator when $\gamma=$ $-a, \omega=1$, and $c=0$ and it reduces to the PSR estimator when $\gamma=-a, \omega=1$, and $c=a v / k$, the PSR estimator dominates the SR estimator. This completes the proof.

\section{Conflict of Interests}

The authors declare that there is no conflict of interests regarding the publication of this paper.

\section{Acknowledgments}

The authors thank the referees and the editors for improving the presentation of the article with some helpful comments and constructive suggestions. This research was partially supported by the Science Foundation of Educational Committee of Jiangxi Province (GJJ12388), the Natural Science Foundation of Jiangxi Province (20122BAB211007), Science and Technology Projection of Hunan (2011GK2009), and the National Social Science Foundation of China (12BTJ014). 


\section{References}

[1] A. Zellner, "Bayesian and non-Bayesian analysis of the regression model with multivariate student- $t$ error terms," Journal of the American Statistical Association, vol. 71, no. 354, pp. 400405, 1976.

[2] C. Stein, "Inadmissibility of the usual estimator for the mean of a multivariate normal distribution," in Proceedings of the Third Berkeley Symposium on Mathematical Statistics and Probability, vol. 1, pp. 197-206, University of California Press, Berkeley, Calif, USA, 1956.

[3] W. James and C. Stein, "Estimation with quadratic loss," in Proceedings of the Fourth Berkeley Symposium on Mathematical Statistics and Probability, Vol. I, pp. 361-379, University of California Press, Berkeley, Calif, USA, 1961.

[4] A. J. Baranchik, "A family of minimax estimators of the mean of a multivariate normal distribution," Annals of Mathematical Statistics, vol. 41, pp. 642-645, 1970.

[5] R. W. Farebrother, "The minimum mean square error linear estimator and ridge regression," Technometrics, vol. 17, pp. 127$128,1975$.

[6] K. Ohtani, "On an adjustment of degrees of freedom in the minimum mean squared error estimator," Communications in Statistics-Theory and Methods, vol. 25, no. 12, pp. 3049-3058, 1996.

[7] J. A. Giles, "Pre-testing for linear restrictions in a regression model with spherically symmetric disturbances," Journal of Econometrics, vol. 50, no. 3, pp. 377-398, 1991.

[8] A. Namba, "PMSE performance of the biased estimators in a linear regression model when relevant regressors are omitted," Econometric Theory, vol. 18, no. 5, pp. 1086-1098, 2002.

[9] A. Namba and K. Ohtani, "Risk comparison of the Stein-rule estimator in a linear regression model with omitted relevant regressors and multivariate $t$ errors under the Pitman nearness criterion," Statistical Papers, vol. 48, no. 1, pp. 151-162, 2007.

[10] A. Zellner, "Bayesian and non-Bayesian estimation using balanced loss functions," in Statistical Decision Theory and Related Topics V, S. S. Gupta and J. O. Berger, Eds., pp. 377-390, Springer, New York, NY, USA, 1994.

[11] J. A. Giles, D. E. A. Giles, and K. Ohtani, "The exact risks of some pre-test and Stein-type regression estimators under balanced loss," Communications in Statistics-Theory and Methods, vol. 25, no. 12, pp. 2901-2924, 1996.

[12] K. Ohtani, D. E. A. Giles, and J. A. Giles, "The exact risk performance of a pre-test estimator in a heteroskedastic linear regression model under the balanced loss function," Econometric Reviews, vol. 16, no. 1, pp. 119-130, 1997.

[13] K. Ohtani, "Inadmissibility of the Stein-rule estimator under the balanced loss function," Journal of Econometrics, vol. 88, no. 1, pp. 193-201, 1999. 


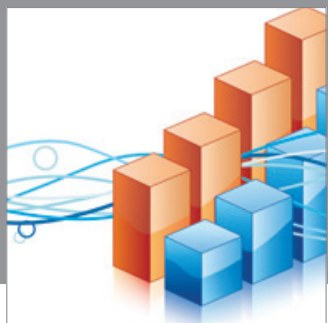

Advances in

Operations Research

mansans

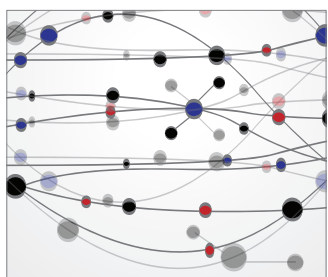

The Scientific World Journal
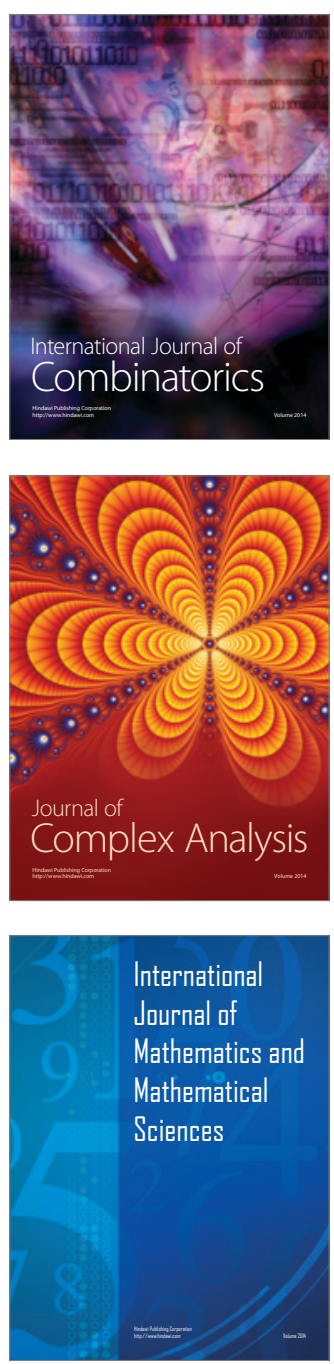
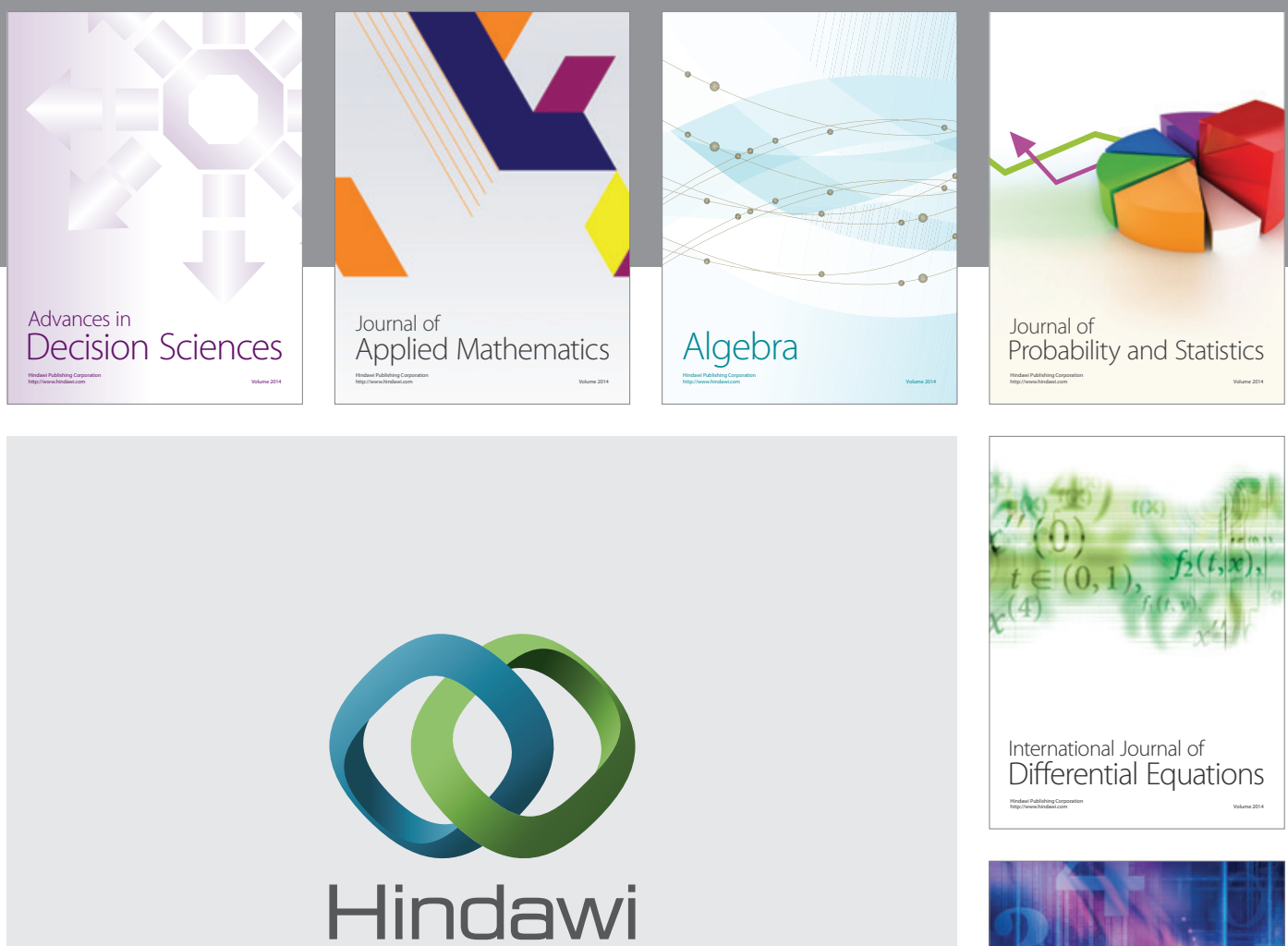

Submit your manuscripts at http://www.hindawi.com
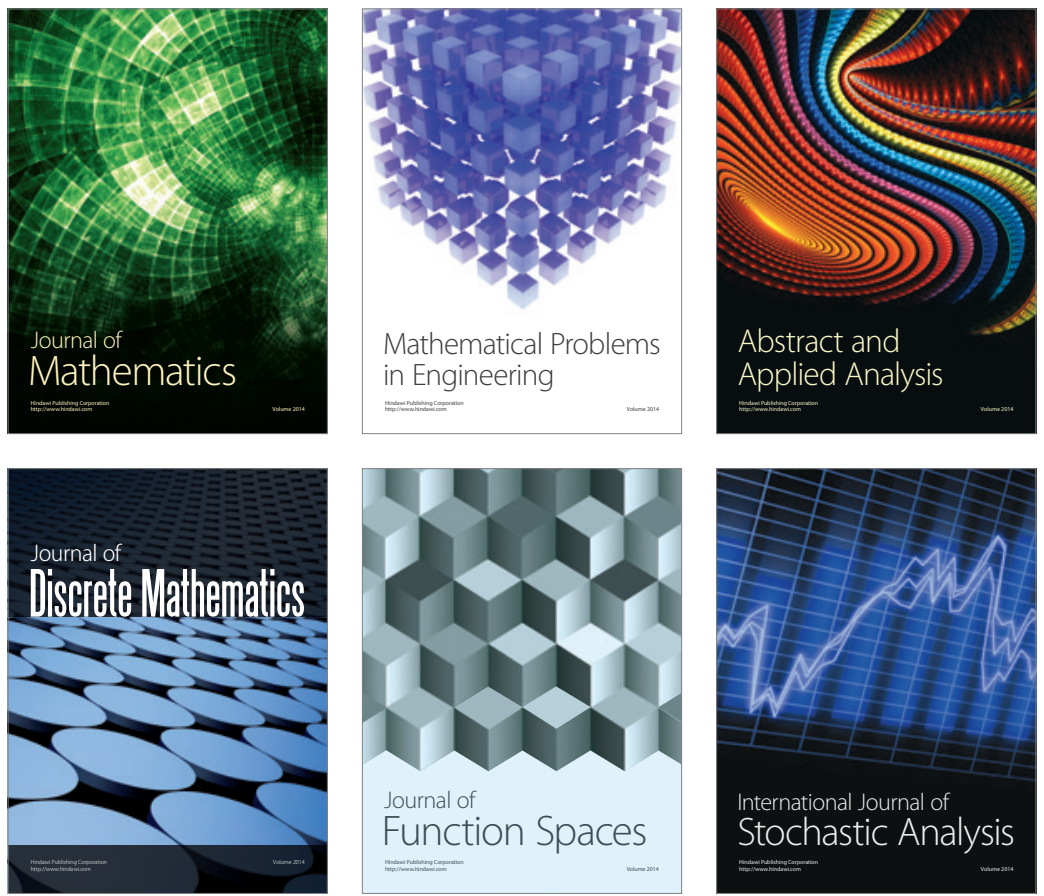

Journal of

Function Spaces

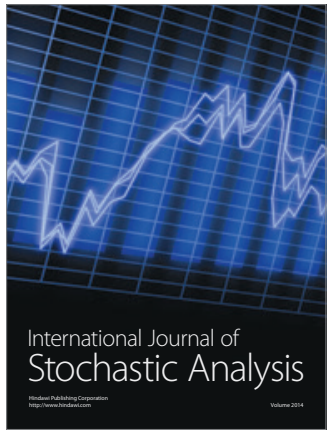

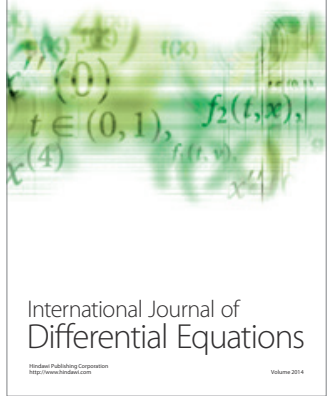
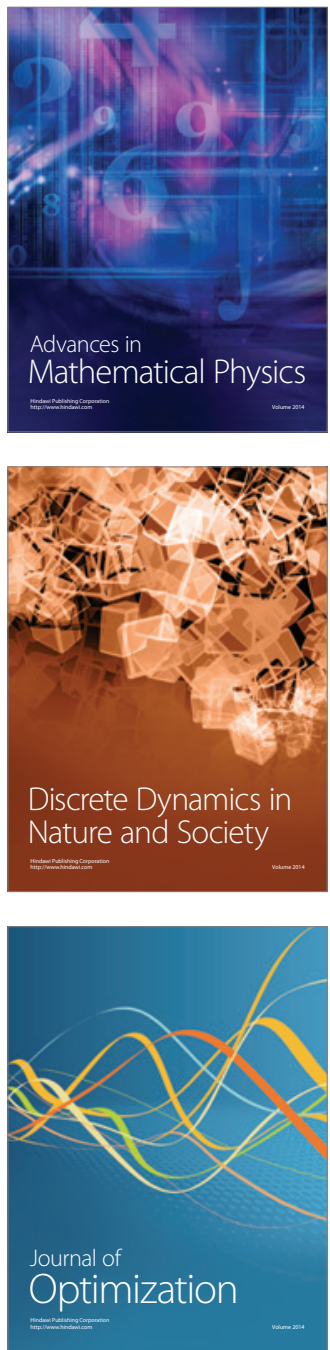